Rev. Adm. Saúde - Vol. 18, № 72, jul. - set. 2018

http://dx.doi.org/10.23973/ras.72.120

ARTIGO DE REVISÃO

\title{
Auditoria nos registros em centro cirúrgico
}

Audit in records in surgical center

\section{Andresa Thomé Silveira ${ }^{1}$, Thiago Thomé Silveira², Patrícia Treviso ${ }^{3}$}

1. Enfermeira, especialista em auditoria em sistemas de saúde. Afiliada ao Centro Educacional São Camilo, Porto Alegre RS

2. Enfermeiro. Enfermeiro do Centro Cirúrgico do Hospital Nossa Senhora da Conceição. Porto Alegre RS

3. Enfermeira. Docente do Curso Enfermagem do Centro Universitário Metodista IPA, Porto Alegre RS

\section{RESUMO}

O presente estudo discute aspectos da auditoria em débitos do centro cirúrgico, tendo como principal objetivo identificar as principais fragilidades e desafios relacionados à auditoria de enfermagem em centro cirúrgico. Trata-se de uma pesquisa bibliográfica que utiliza, como corpo de análise, artigos redigidos em português, inglês e espanhol. Publicados em periódicos indexados nas bases de dados LILACS, BDENF, IBECS, SciELO, MEDLINE e PubMed, que se enquadraram nos critérios de inclusão. Para a seleção das publicações foram utilizadas as técnicas de leitura exploratória, seletiva, analítica e interpretativa. Foram analisados 05 artigos, cuja leitura interpretativa resultou na organização dos dados em 03 categorias: (1) Falha nos Registros; (2) Educação Continuada e (3) Recursos Humanos de Enfermagem. Percebe-se que o problema de falhas nos registros foi citado em todos os artigos selecionados. A educação continuada, vem ao encontro favorecendo a qualidade dos registos e mantendo a equipe atualizada para 0 atendimento adequado. A questão de recursos humanos de enfermagem foi citada somente em um dos artigos, onde relatam que os eventos adversos podem estar relacionados com a falta de tempo e todas as atividades que os profissionais necessitam desempenhar. Conclui-se com este estudo que é importante estimular o aprofundamento de pesquisas 
sobre esta temática, com o intuito de aprimorar os registros para se alcançar melhores resultados assistenciais e, também, financeiros para as instituições envolvidas neste processo, garantindo sempre a qualidade do atendimento prestado.

Palavras-chave: Auditoria de Enfermagem; Enfermagem de Centro Cirúrgico; Enfermagem

\section{ABSTRACT}

The present study discusses aspects of auditing debts of the surgical center, with the main objective to identify as main fragilities and challenges related to nursing care in a surgical center. This is a bibliographical research that uses, as analysis body, articles written in Portuguese, English and Spanish. Published in journals indexed in the databases LILACS, BDENF, IBECS, SciELO, MEDLINE and PubMed, which were included in the inclusion criteria. For the selection of the publications the exploratory, selective, analytical and interpretive reading techniques were used. We analyzed 05 articles, whose interpretive reading resulted in the organization of the data in 03 categories: (1) Records Failure; (2) Continuing Education and (3) Nursing Human Resources. It is noticed that the problem of record failures was quoted in all selected articles. Continuing education comes to the meeting favoring the quality of the records and keeping the team up to date for the appropriate service. The issue of nursing human resources was cited only in one of the articles, where they report that adverse events may be related to the lack of time and all the activities that professionals need to perform. It is concluded with this study that it is important to stimulate the deepening of research on this subject, with the intention of improving the records to achieve better healthcare and financial results for the institutions involved in this process, always guaranteeing the quality of care provided.

Keywords: Nursing Audit; Operating Room Nursing; Nursing.

\section{INTRODUÇÃO}

O período de turbulência política e econômica em que o país vive nos dias atuais, exige das instituições de saúde não só contenção de recursos, mas um rigoroso monitoramento e avaliação de seus processos ${ }^{1}$.

A auditoria em débitos tem sido cada vez mais criteriosa. Sabe-se que se os registros se tornarem totalmente corretos, a instituição não só diminuirá perdas, como também obterá resultados positivos, melhorando a gestão dos processos $^{1}$. A auditoria em débitos é de suma importância, pois aponta as irregularidades dos registros, evitando perdas na receita as quais têm como consequência o aumento dos custos para a instituição. 
Com a evolução do cenário epidemiológico nacional e o crescente desenvolvimento técnico-científico, paralelamente à competitividade entre as instituições hospitalares, os serviços de saúde passaram a oferecer tratamentos cada vez mais dispendiosos, gerando preocupação desses prestadores em otimizar seus custos, minimizar as perdas e avaliar a assistência ${ }^{2}$. Nesse contexto, a auditoria de enfermagem trabalha ativamente na busca de melhorias dos processos e na redução dos custos.

A auditoria de enfermagem é uma avaliação sistemática, realizada através dos registros de enfermagem no prontuário do paciente, e tem como uma de suas finalidades fornecer dados para a melhoria da qualidade do cuidado e melhoria dos próprios registros ${ }^{3}$, além de contribuir, também, para a melhoria dos processos e redução de custos. Segundo Arantes, Helito e Silva ${ }^{4}$, são fundamentais para um enfermeiro auditor e seu desenvolvimento profissional o conhecimento técnico-científico e uma visão holística e generalista dos processos assistenciais e administrativos.

A auditoria é peça chave na conferência dos registros e cobrança das contas, mas o trabalho que a auditoria realiza dentro de uma instituição de saúde é uma atividade que vai além de uma simples conferência de compatibilidade entre os procedimentos realizados e a cobrança hospitalar ${ }^{5}$. Nesse contexto, a enfermagem possui uma visão ampla do funcionamento das instituições hospitalares, assim facilitando a sua atuação para esta função.

A enfermagem pode atuar nas duas modalidades de auditoria, sendo estas: auditoria retrospectiva, após alta do paciente ou na auditoria concorrente, enquanto o paciente está hospitalizado ou em atendimento ${ }^{3}$.

$\mathrm{Na}$ área de centro cirúrgico, o auditor de enfermagem atua também na elaboração de pacotes de procedimentos cirúrgicos, buscando melhorar a negociação do hospital com as operadoras de planos de saúde 5 . De acordo com o mesmo autor, o enfermeiro auditor precisa conhecer e dominar todos os processos que envolvem o atendimento do paciente, desde a entrada deste na instituição, até a sua saída.

Os registros de enfermagem devem abranger as informações sobre a saúde geral do paciente, sobre as observações e intervenções realizadas, bem como acolher as informações administrativas referentes a transferências, equipamentos e insumos ${ }^{6}$. Nessa área, o enfermeiro pode atuar não apenas como conferente de materiais e medicamentos, mas tem condições, também, de sugerir estratégias e mudanças das rotinas, subsidiando a educação continuada com foco na prestação de serviços de qualidade e no controle das perdas $^{2}$.

Outrossim, os registros de enfermagem são necessários para a realização das auditorias, e para respaldo ético e legal perante o conselho e a justiça.

Ressalta-se que a anotação de enfermagem não deve ser encarada apenas como um cumprimento de normas, passível de esquecimento. Pelo contrário, é preciso que se tenha uma noção da sua real importância e das implicações decorrentes do registro incorreto ${ }^{7}$. 
O centro cirúrgico é uma unidade diferenciada, de elevada complexidade, devido às suas particularidades e características. Em virtude disso, possui equipamentos de alta tecnologia e necessita de profissionais qualificados ${ }^{8}$. $O$ cenário da saúde está em constante evolução, e o centro cirúrgico é uma das unidades que se atualiza permanentemente nos aspectos técnicos e operacionais para dispor de um atendimento de qualidade ${ }^{9}$.

Vale ressaltar que as novas tecnologias trazem ao profissional enfermeiro novos desafios em seu processo de trabalho, já que todas essas evoluções exigem desenvolvimento de novas habilidades, outra destreza e aperfeiçoamento progressivo e contínuo ${ }^{10}$.

Os custos hospitalares têm aumentado consideravelmente de ano a ano devido à complexidade de tratamentos e procedimentos realizados, agregada ao alto valor dos materiais e medicamentos modernos da área cirúrgica ${ }^{11}$. E a falta de controle dos gastos e dos recursos utilizados em cirurgia tem contribuído para 0 aumento de custos dos procedimentos ${ }^{12}$. Nesse cenário, a auditoria em contas hospitalares possibilita uma análise precisa das cobranças e facilita a informação efetiva dos dados apresentados ${ }^{13}$.

Assim, o enfermeiro emerge como um profissional que tem competência e conhecimento para atuar no processo de auditoria dos registros do centro cirúrgico. No entanto, muitos ainda são os desafios que permeiam esses processos. Diante disso, questiona-se: quais os desafios relacionados à auditoria de centro cirúrgico, descritos na literatura?

Isso posto, o objetivo deste estudo é identificar as fragilidades, já apontadas em outros estudos, para categorizá-las, configurando-as como desafios relacionados à auditoria de enfermagem realizada em registros de centro cirúrgico.

\section{MÉTODOS}

Trata-se de um estudo de revisão bibliográfica. Tal metodologia propõe o estabelecimento de critérios bem definidos quanto à coleta de dados, análise e interpretação dos resultados. Seis etapas foram adotadas para a realização deste estudo: 1) construção da questão de pesquisa; 2) definição dos critérios de inclusão e exclusão dos artigos e determinação da amostra; 3 ) representação dos estudos selecionados em formato de tabelas, considerando todas as características em comum; 4) análise crítica dos resultados, identificando diferenças e conflitos; 5) interpretação dos dados; e 6) relato, de forma clara, da evidência encontrada.

A estratégia de identificação e seleção dos estudos fundamentou-se em publicações indexadas nas bases de dados Literatura Latino-Americana e do Caribe em Ciências da Saúde (LILACS), Base de Dados Bibliográficos Especializada na Área de Enfermagem do Brasil (BDENF), Índice Bibliográfico Español em Ciencias de la Salud (IBECS), Scientific Eletronic Library Online (SciELO), Sistema Online de Busca e Análise de Literatura Médica (MEDLINE) 
e PubMed, consideradas como as principais bases de dados das ciências da saúde em geral e enfermagem brasileira.

A busca das informações nas bases de dados ocorreu a partir dos descritores classificados nos Descritores em Ciências da Saúde (DeCS): auditoria de enfermagem; enfermagem de centro cirúrgico e enfermagem. A utilização dos operadores booleanos "[AND]" e "[OR]" permitiu a realização de uma busca avançada com os descritores.

Foram adotados os seguintes critérios de inclusão: artigos publicados em português, inglês e espanhol, disponíveis na íntegra online e gratuitos, de 2001 a 2016, relacionados com a questão norteadora da pesquisa. Excluíram-se artigos incompletos, indisponíveis, publicações repetidas, artigos em outra língua, teses, dissertações, trabalhos de conclusão de curso, manuais e livros ou os que não tinham relação com o objetivo proposto.

Após identificação nas bases de dados, resultou na seleção de 334 referências. Destas, 150 publicações foram excluídas por não compreenderem o período estipulado, 11 publicações foram excluídas por motivo de duplicidade, restando 173 para seleção das análises dos títulos e resumos relacionando os artigos com o problema e o objetivo da pesquisa. Após esta etapa foram selecionados 24 artigos, destes 11 enquadravam-se nos critérios de exclusão.

Desta forma, 13 artigos foram submetidos a leitura na integra pelos pesquisadores, destes, 5 artigos constituem o corpus de análise do estudo (Figura 1).

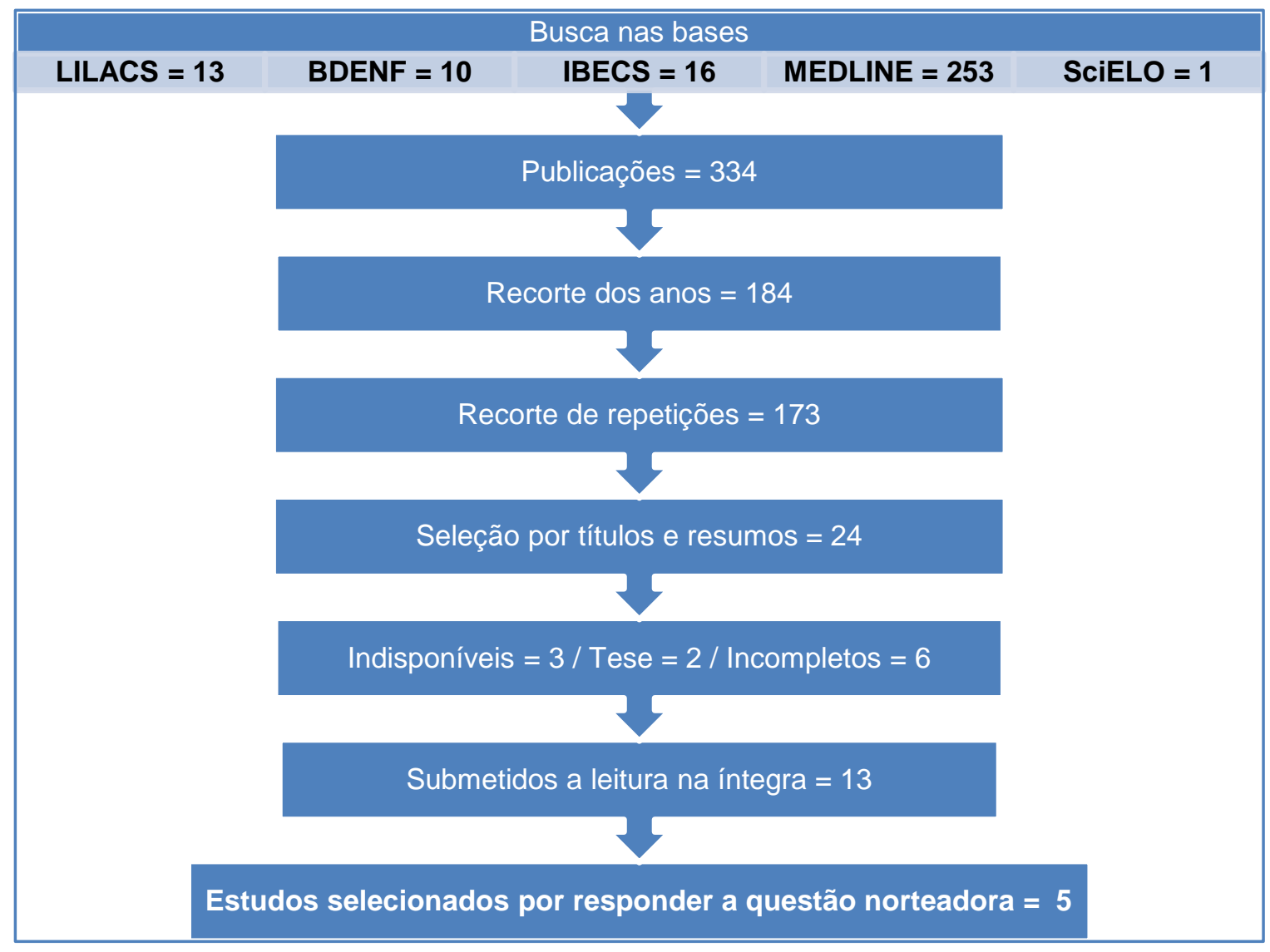


Figura 1. Fluxograma de seleção dos estudos.

Os artigos foram rigorosamente referenciados, sendo respeitadas as ideias dos autores, conforme previsto na Lei $n^{-} 12.853 / 13$, Lei do Direito Autoral ${ }^{14}$. Sendo norteado pelas normas ABNT.

\section{RESULTADOS E DISCUSSÃO}

O corpus de análise da pesquisa é constituído por 5 artigos publicados no período de 2001 a 2016. Desses artigos, evidenciou-se a LILACS como a base de dados com mais publicações sobre o tema (40\%). O maior número de publicações ocorreu no ano de 2011 (40\%). Quanto à distribuição dos artigos por nacionalidade, todos são brasileiros (100\%), com maior concentração na região sudeste $(40 \%)$.

No que se refere às metodologias utilizadas, observa-se o predomínio da abordagem descritiva, retrospectiva, quantitativa (40\%) e relato de experiência $(40 \%)$, seguida do estudo qualitativo, descritivo, exploratório (20\%). Todos os estudos analisados foram realizados em hospitais (100\%). O Quadro 1 apresenta a síntese dos artigos selecionados. 
Quadro 1. Síntese dos artigos selecionados, de acordo com ano, autor(es), título, publicação e categoria.

\begin{tabular}{|c|c|c|c|c|c|}
\hline $\begin{array}{l}\text { Número } \\
\text { de } \\
\text { ordem }\end{array}$ & Ano & Autor & Título & Publicação & $\begin{array}{l}\text { Categoria em } \\
\text { que se insere }\end{array}$ \\
\hline $\mathrm{A} 1$ & 2016 & $\begin{array}{l}\text { Souza MPD, } \\
\text { Ceretta } \\
\text { LB,Soratto, MT. }\end{array}$ & $\begin{array}{l}\text { Auditoria } \\
\text { concorrente no } \\
\text { centro cirúrgico: } \\
\text { concepções dos } \\
\text { enfermeiros. }\end{array}$ & $\begin{array}{l}\text { Revista Saúde } \\
\text { e Pesquisa } \\
\text { (v.9, n.2, } \\
\text { p.263-272). }\end{array}$ & $\begin{array}{l}\text { Falha nos } \\
\text { registros; } \\
\text { Educação } \\
\text { continua; }\end{array}$ \\
\hline $\mathrm{A} 2$ & 2009 & $\begin{array}{l}\text { Setz VG, } \\
\text { D'Innocenzo M. }\end{array}$ & $\begin{array}{l}\text { Avaliação da } \\
\text { qualidade dos } \\
\text { registros de } \\
\text { enfermagem no } \\
\text { prontuário por } \\
\text { meio de auditoria. }\end{array}$ & $\begin{array}{l}\text { Acta Paulista } \\
\text { de } \\
\text { Enfermagem } \\
\text { (v.22, n.3, } \\
\text { p.313-317). }\end{array}$ & $\begin{array}{l}\text { Falha nos } \\
\text { registros }\end{array}$ \\
\hline A3 & 2013 & $\begin{array}{l}\text { Oliveira DR, } \\
\text { Jacinto SM, } \\
\text { Siqueira CL }\end{array}$ & $\begin{array}{l}\text { Auditoria de } \\
\text { enfermagem em } \\
\text { centro cirúrgico. }\end{array}$ & $\begin{array}{l}R A S \text { (v. 15, n. } \\
61, p 151-158)\end{array}$ & $\begin{array}{l}\text { Falha nos } \\
\text { registros; } \\
\text { Educação } \\
\text { continuada; } \\
\text { Recursos } \\
\text { humanos de } \\
\text { enfermagem; }\end{array}$ \\
\hline A4 & 2011 & $\begin{array}{l}\text { Medrado SDSR, } \\
\text { Moraes, MWD }\end{array}$ & $\begin{array}{l}\text { Auditoria de } \\
\text { enfermagem em } \\
\text { centro cirúrgico: } \\
\text { atuação do } \\
\text { enfermeiro } \\
\text { auditor. }\end{array}$ & $\begin{array}{l}\text { Revista } \\
\text { SOBECC } \\
\text { (v.16, n.1, p56- } \\
62)\end{array}$ & $\begin{array}{l}\text { Falha nos } \\
\text { registros; } \\
\text { Educação } \\
\text { continuada; }\end{array}$ \\
\hline A5 & 2011 & $\begin{array}{l}\text { Sousa PV, } \\
\text { Passos KFDM, } \\
\text { Torres LCGFL, } \\
\text { Mulatinho LM }\end{array}$ & $\begin{array}{l}\text { Auditoria em } \\
\text { enfermagem: } \\
\text { uma contribuição } \\
\text { à minimização } \\
\text { das glosas } \\
\text { hospitalares. }\end{array}$ & $\begin{array}{l}\text { Revista } \\
\text { Enfermagem } \\
\text { UFPE On Line } \\
\text { (v.5, n.10, p. } \\
2479-2483) .\end{array}$ & $\begin{array}{l}\text { Falha nos } \\
\text { registros; } \\
\text { Educação } \\
\text { continuada; }\end{array}$ \\
\hline
\end{tabular}

A partir da análise do quadro, observa-se que todos os artigos publicados foram em periódicos diferentes (100\%), sendo que $60 \%$ foram publicados em periódicos de enfermagem e os demais $(40 \%)$ em periódicos de outras áreas. Em relação ao ano de publicação, os artigos se concentram no período de 2009 a 2016.

Numa leitura analítica dos artigos, verificou-se a incidência de alguns dados que foram agrupados, tendo em vista a formação de categorias. Com a 
finalidade de sistematizar para melhor discutir esses dados estabeleceram-se três categorias: falha nos registros de enfermagem; educação continuada e recursos humanos de enfermagem.

\section{Falha nos Registros}

Esta categoria foi discutida em todos os artigos analisados (A1, A2, A3, A4, A5). Todos eles evidenciam a falha nos registros como um dos principais desafios para a auditoria de contas hospitalares.

No processo de atenção à saúde, os registros das atividades realizadas constituem um dos instrumentos efetivos para a comunicação e o planejamento dos serviços prestados ao cliente ${ }^{15}$. Todos os medicamentos e materiais utilizados em cirurgia precisam ser devidamente preenchidos e checados com início e término, sendo que nas instituições em que ainda não se utilizam checagem digital, o registro deve ser feito de forma legível com suas respectivas quantidades, compatíveis com as fichas de descrições ${ }^{5}$. É necessário também controlar o uso adequado destes materiais para que não haja desperdícios, pois neste setor utilizam-se materiais de alto custo.

O artigo A5 mostra que é alto o percentual de glosas devido às falhas das anotações de enfermagem, principalmente com materiais e medicamentos, representando um valor alto de perda para a instituição. Assim que todos os materiais e medicamentos utilizados nos procedimentos com os pacientes devem estar devidamente preenchidos e checados $^{5}$, a fim de não ter erro no fechamento da conta hospitalar.

Os artigos A2 e A4 relatam problemas nos registros de enfermagem e da equipe médica, tais como: ilegibilidade, informações incompletas, erros de ortografia, falta de checagem e de justificativas, tornando, assim, um risco para o profissional e sua instituição. Analisando todos os aspectos dos registros, pode-se assim caracterizá-los: eles devem ser legíveis, de preenchimento correto e claro e com descrição de toda a assistência prestada ao cliente durante sua passagem dentro de uma instituição ${ }^{5}$. 0 não registro, ou o registro incorreto, dificulta o reconhecimento do gasto real de cada cirurgia e, consequentemente, perde-se o controle de perdas e geram-se desperdícios que possam estar ocorrendo ${ }^{12}$.

O artigo A3 relata que as anotações incompletas desproporcionam subsídios para a análise da auditoria de enfermagem interferindo diretamente na qualidade do serviço prestado. No cotidiano a enfermagem desempenha suas atividades de maneira organizada, mas quando falta sistematização destas atividades, a continuidade e a qualidade da assistência podem estar prejudicadas $^{16}$.

As falhas ocorridas, na maioria das vezes por falta de registros, acabam elevando as glosas e acarretando, de forma expressiva, as despesas da instituição e a auditoria pode desempenhar um papel proativo importante neste aspecto, evitando o retrabalho (A1). Paralelamente, os mesmos autores 
relatam que a auditoria concorrente pode beneficiar e agilizar o controle destas atividades, otimizando os recursos e visando a melhoria contínua.

Na prática da assistência, a comunicação escrita tem sido negligenciada pelos profissionais de enfermagem, pois os registros, quando realizados, são escassos e incompletos ${ }^{17}$. Embora os enfermeiros saibam dessa importância, muitas vezes não conseguem efetivar esta prática, de forma satisfatória, no cotidiano (A3)

\section{Educação continuada}

O artigo A4 aponta a importância da educação continuada, buscando o registro adequado dos processos e insumos que geram custos à instituição. O estudo ainda aponta que as capacitações, de modo geral, são focadas na assistência e não nos processos administrativos. Vale ressaltar que os processos administrativos e burocráticos impactam na sustentabilidade da instituição, exigindo, portanto, registros corretos e fidedignos, possibilitando a auditoria e, por conseguinte, benefícios à instituição.

Faz-se necessária a realização de treinamentos sistematizados para capacitar a equipe de enfermagem, na busca da valorização das anotações dos profissionais de saúde, da melhoria nos resultados e do monitoramento dos cuidados prestados ${ }^{6}$.

Evidenciou-se que a falha constante nos registros é uma das principais causas da perda de receita. Os autores do artigo A5 descrevem que a educação continuada contribui para a diminuição das glosas e o aumento do faturamento do hospital. Desta forma, a educação continuada quando implantada no cotidiano da prática de enfermagem como forma de crescimento, aperfeiçoamento e sugestões possibilita na solução dos problemas nos registros $^{18}$.

Nos artigos A1 e A3, a educação continuada serve para orientar a equipe multidisciplinar para executar os registros de forma adequada e completa, ao mesmo tempo, compartilha todas as contas que apresentam algum problema, desta forma evitam falhas no processo e agilizam a fatura. Ressalta-se que a auditoria deve ocorrer em caráter educativo, promovendo ações educativas diante dos fatos, não apontando culpados e sim investigando as causas dos acontecimentos e, desta forma, atuando na melhoria dos processos ${ }^{6}$, e capacitando de forma continuada, tanto a equipe assistencial como a equipe administrativa.

O processo de trabalho do enfermeiro permeia as esferas gerencial e assistencial, perpassando o ensino e a pesquisa, que estão diretamente ligados às práticas do cuidado, tornando-o, dessa forma, um gerenciador do cuidado ${ }^{19}$.

\section{Recursos Humanos de Enfermagem}


No que se refere aos recursos humanos de enfermagem, o artigo A3 pontua que o quantitativo de recursos humanos influência na ocorrência de falhas nos registros, visto que, nesse cenário, a equipe prioriza a assistência ao invés dos registros, por não haver tempo hábil suficiente para desempenhar ambas tarefas.

Para viabilizar a assistência de enfermagem é necessário ter uma estrutura de recursos humanos em quantidade, composição, distribuição adequada por turnos e setores dentro das instituições ${ }^{20}$. O dimensionamento dos recursos humanos de enfermagem constitui na etapa inicial do processo de provimento de pessoal e tem, por finalidade, a previsão da quantidade suficiente de funcionários para atender às necessidades dos clientes ${ }^{21}$.

Historicamente, a equipe de enfermagem sofre com a carência de contingente adequado para a realização de uma segura assistência de qualidade. $O$ Conselho Federal de Enfermagem publicou em 2017 a Resolução n. $543{ }^{22}$ que atualiza e estabelece parâmetros para o dimensionamento do quadro de profissionais de enfermagem nos serviços/locais em que são realizadas atividades de enfermagem. Entretanto, mesmo com os esforços dispendidos para adequação desses parâmetros, a realidade enfrentada por muitos profissionais, em diversas instituições, ainda é de grande sobrecarga de trabalho devido ao dimensionamento inadequado.

\section{CONSIDERAÇÕES FINAIS}

O estudo possibilitou evidenciar, de forma sistemática, que os maiores desafios no processo de auditoria de enfermagem de centro cirúrgico envolvem falhas nos registros, falta de capacitação sobre o que deve ser registrado e de consciência sobre a importância dos registros, como também dimensionamento de profissionais de enfermagem.

As instituições de saúde, de modo geral, buscam a qualidade nos serviços prestados, sem perder o foco da sustentabilidade, pois lidam com um pensamento empresarial, requerendo associar baixos custos com a concorrência e excelência no atendimento prestado. Para isso, necessitam investir constantemente na qualificação e conscientização de seus profissionais, visando boas práticas assistenciais e administrativas, que possibilitem, dessa forma, a prestação de serviços qualificados, garantindo resultados positivos e clientes satisfeitos.

Nesse cenário, destaca-se a importância do papel do auditor de enfermagem, na diminuição dos custos e na busca por melhores resultados para as instituições hospitalares e para os usuários de seus serviços.

\section{REFERÊNCIAS}


1. Azevedo GA, Gonçalves NS, Santos DC. A relação entre a auditoria e o sistema público em saúde. Rev. Adm. Saúde [Internet]. 2018 [acesso em 2018 abr.];18(70). Doi: http://dx.doi.org/10.23973/ras.70.91

2. Scarparo AF. Auditoria em enfermagem: revisão de literatura. Nursing (São Paulo). 2005;80(8):46-50.

3. Pereira LL, Takahashi RT. Auditoria em enfermagem. In: Kurcgant P. Administração em enfermagem. São Paulo: EPU; 1991. p. 215-222.

4. Arantes SC, Helito RAB, Silva LR. Auditoria de enfermagem em contas hospitalares. In: D'Innocenzo M. Indicadores, auditorias, certificações: ferramentas de qualidade para gestão em saúde. $2^{a}$ ed. São Paulo: Martinari; 2010. p. 127-133.

5. Motta ALC. Auditoria de enfermagem nos hospitais e operadoras de planos

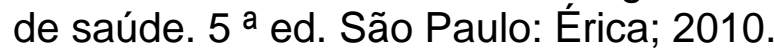

6. Labbadia LL. Registros de enfermagem: avaliação e critérios. In: D'Innocenzo M. Indicadores, auditorias, certificações: ferramentas de qualidade para gestão em saúde. $2^{\underline{a}}$ ed. São Paulo: Martinari; 2010. p.161165.

7. Ferreira TS, de Souza-Braga AL, Cavalcanti-Valente GS, Ferreira de Souza D, Carvalho-Alves EM. Auditoria de enfermagem: o impacto das anotações de enfermagem no contexto das glosas hospitalares. Aquichán. 2009;9(1):38-49.

8. Duarte IG, Ferreira DP. Uso de indicadores na gestão de um centro cirúrgico. Rev. Adm. Saúde. 2006;8(31):63-70.

9. Akeme J. Gerenciamento em centro cirúrgico. In: Malagutti W, Bonfim IM. Enfermagem em centro cirúrgico: atualidades e perspectivas no ambiente cirúrgico. São Paulo: Martinari; 2008. p. 57-68.

10. Pinto EV, Treviso P, Botene DZA. Atuação do enfermeiro na cirurgia robótica: desafios e perspectivas. Rev SOBECC. 2018;23(1):43-51.

11. Castilho V, Leite MMJ. A Administração de recursos materiais na enfermagem. In: Kurcgant P. Administração em enfermagem. São Paulo: EPU; 1991. p. 73-76.

12. Bittar E, Castilho V. Análise de preenchimento da ficha de gastos de material de consumo em cirurgia de revascularização do miocárdio: dificuldades e sugestões. Cad. Centro Universitário São Camilo. 2003;9(3):68-77.

13. Buzatti CV, Chianca TC. Auditoria em enfermagem: erros e custos envolvidos nas anotações. Nursing (São Paulo). 2005;90(8):518-22.

14. Brasil. Lei $n^{\circ} 12.853$, de 14 de agosto de 2013 [Internet]. Altera os arts. $5^{\circ}$, 68, 97, 98, 99 e 100, acrescenta arts. 98-A, 98-B, 98-C, 99-A, 99-B, 100-A, 
100-B e 109-A e revoga o art. 94 da Lei o 9.610, de 19 de fevereiro de 1998, para dispor sobre a gestão coletiva de direitos autorais, e dá outras providências. Brasília; 2013 ago. 13 [acesso em 2018 jul.]. Disponível em: http://www.planalto.gov.br/ccivil_03/_Ato2011-2014/2013/Lei/L12853.htm

15. Matsuda LM, Carvalho ARS, Évora YDM. Anotações/registros de enfermagem em um hospital escola. Rev Cienc Cuidad. 2007;6(2):337-46.

16. Guido LA, Goulart CT, Brum CN, Lemos, AP, Umman J. Cuidado de enfermagem perioperatório: revisão integrativa de literatura. Rev Pesqui Cuid Fundam. (Online). 2014;6(4):1601-9. doi:

http://dx.doi.org/10.9789/2175-5361.2014.v6i4.1601-1609

17. Pimpão FD, Lunardi Filho WD, Vaghetti HH, Lunardi VL. Percepção da equipe de enfermagem sobre seus registros: buscando a sistematização da assistência de enfermagem. Rev Enferm UERJ. 2010 jul/set;18(3):405-10.

18. Luz AM, Martins AP, Dynewicz AM. Características de anotações de enfermagem encontradas em auditoria. Rev Eletrônica Enferm [Internet]. 2007 [acesso em 2018 abr.];9(2):344-61. Disponível em: https://www.revistas.ufg.br/fen/article/viewFile/7165/5074. doi: https://doi.org/10.5216/ree.v9i2.7165

19. Treviso P, Peres SC, Silva AD, Santos AA. Competências do enfermeiro na gestão do cuidado. Rev. Adm. Saúde [Internet]. 2017 [acesso em 2018 abr];17(69). Disponível em: http://www.cqh.org.br/ojs2.4.8/index.php/ras/article/view/59. doi: http://dx.doi.org/10.23973/ras.69.59

20. D'Innocenzo M. Indicadores de recursos humanos. In: D'Innocenzo M. Indicadores, auditorias, certificações: ferramentas de qualidade para gestão em saúde. $2^{\mathrm{a}}$ ed. São Paulo: Martinari; 2010. p.153-155.

21. Gaidzinski RR. Dimensionamento de pessoal de enfermagem. In: Kurcgant P. Administração em enfermagem. São Paulo: EPU; 1991. p. 91-93.

22. Conselho Federal de Enfermagem (BR). Resolução COFEN № 543/2017, de 18 de abril de 2017. Atualiza e estabelece parâmetros para o Dimensionamento do Quadro de Profissionais de Enfermagem nos serviços/locais em que são realizadas atividades de enfermagem. Brasília (DF): COFEN; 2017.

Recebido: 30 de julho de 2018. Publicado: 20 de setembro de 2018

Correspondência: Patrícia Treviso. E-mail: ptreviso15@gmail.com 
Conflito de Interesses: os autores declararam não haver conflito de interesses.

(C) This is an Open Access article distributed under the terms of the Creative Commons Attribution License, which permits unrestricted use, distribution, and reproduction in any medium, provided the original work is properly cited 